\title{
Preventing atrial fibrillation by combined right isthmus ablation and cryoballoon pulmonary vein isolation in patients with typical atrial flutter: PAF-CRIOBLAF study
}

\author{
Frédéric Anselme MD, $\mathrm{PhD}^{1}$ (D) | Arnaud Savouré $\mathrm{MD}^{1}$ | Nicolas Clémenty MD, $\mathrm{PhD}^{2}$ | \\ Olivier Cesari MD ${ }^{3}$ | Dominique Pavin MD ${ }^{4}$ Laurence Jesel MD, PhD ${ }^{5}$ | Pascal Defaye \\ $M^{6}$ | Serge Boveda $M^{7}$ | Philippe Rivat $M^{8}$ | Jacques Mansourati MD, $\mathrm{PhD}^{9}$ | \\ Alexis Mechulan MD ${ }^{10}$ | Jean-Pierre Cebron MD $^{11}$ | Gilles Lande MD ${ }^{12}$ | \\ Michael Bubenheim ScD ${ }^{13}$ | Antoine Milhem MD ${ }^{14}$
}

${ }^{1}$ Department of Cardiology, Rouen University Hospital, Rouen, France

${ }^{2}$ Department of Cardiology, Tours University Hospital, Tours, France

${ }^{3}$ Department of Cardiology, Clinique SaintGatien, Tours, France

${ }^{4}$ Department of Cardiology, Rennes University Hospital, Rennes, France

${ }^{5}$ Department of Cardiology, Strasbourg University Hospital, Strasbourg, France

${ }^{6}$ Department of Cardiology, Grenoble- Alpes University Hospital, Grenoble, France

${ }^{7}$ Department of Cardiology, Clinique Pasteur, Toulouse, France

${ }^{8}$ Department of Cardiology, Polyclinique

Vauban, Valenciennes, France

${ }^{9}$ Department of Cardiology, Brest University Hospital, Boulevard Tanguy Prigeant, Brest, France

${ }^{10}$ Department of Cardiology, Hôpital privé de Clairval, Marseille, France

${ }^{11}$ Department of Cardiology, Nouvelles Cliniques Nantaises, Nantes, France

${ }^{12}$ Department of Cardiology, Nantes University Hospital, Nantes, France

${ }^{13}$ Department of Biostatistics, Rouen University Hospital, Rouen, France

${ }^{14}$ Department of Cardiology, Centre hospitalier de La Rochelle, La Rochelle, France

\section{Correspondence}

Frédéric Anselme, Service de Cardiologie, Hôpital Charles Nicolle, 1 rue de Germont,

\begin{abstract}
Background: Although less common, typical atrial flutter shares similar pathophysiological roots with atrial fibrillation. Following successful cavo-tricuspid isthmus ablation using radiofrequency, many patients, however, develop atrial fibrillation in the mid-to-long-term. This study sought to assess whether pulmonary vein isolation conducted at the same time as cavo-tricuspid isthmus ablation would significantly modify the atrial fibrillation burden upon follow-up in patients suffering from typical atrial flutter.

Methods: This was a multicenter randomized controlled study involving typical atrial flutter patients with history of non-predominant atrial fibrillation (1 atrial fibrillation episode only, in $67 \%$ of population) who were scheduled for cavo-tricuspid isthmus radiofrequency ablation. Patients were randomly assigned to either undergo cavotricuspid isthmus ablation alone or cavo-tricuspid isthmus plus pulmonary vein isolation $(\mathrm{CTI}+)$. Pulmonary vein isolation was performed using cryoballoon technology. An outpatient consultation with ECG and 1-week Holter monitoring was performed at 3, 6 months, 1 year, and 2 years postprocedure. The primary endpoint was atrial fibrillation recurrences lasting more than $30 \mathrm{~s}$ at 2 years postablation.

Results: Of the patients enrolled, 36 were included in each group. At 2-year follow-up, the atrial fibrillation recurrence rate was significantly higher in the CTI vs $\mathrm{CTI}+$ group (25/36, $69 \%$ vs. $12 / 36,33 \%$ respectively; $P<.001)$, with similar typical atrial flutter recurrence rates. There were no differences in undesirable events, except for transient phrenic nerve palsy reported from three $\mathrm{CTI}+$ patients (8.3\%).

Conclusion: Pulmonary vein isolation using cryoballoon technology was proven to significantly reduce the atrial fibrillation incidence at 2 years postcavo-tricuspid isthmus ablation.
\end{abstract}


FR-76031 Rouen, France.

Email: frederic.anselme@chu-rouen.fr

Funding information

Medtronic

\section{KEYWORDS}

ablation techniques, atrial fibrillation, atrial flutter, cryoablation, pulmonary veins

\section{1 | INTRODUCTION}

Though typical atrial flutter (AFL) is less common than atrial fibrillation (AF), both arrhythmias likely share similar pathophysiological triggers. Despite the high success rates of AFL ablation, ${ }^{1,2}$ patients are nevertheless at risk of developing AF postablation, the incidence of which has been reported to be as high as $50 \%$ or even higher. ${ }^{3}$ Moreover, it must be stressed that the thromboembolic risk of AFL patients has been primarily linked to $A F$ occurrence and the underlying cardiopathy. ${ }^{4} \mathrm{AF}$ ablation using cryoballoon technology to isolate pulmonary veins has been reported to be as efficient as ablation by means of RF in managing paroxysmal AF, with very few complications reported worldwide. ${ }^{5}$

Considering the performance and safety profile of cryoballoons, along with the high-risk of AFL patients to develop AF after successful AFL ablation, we designed and implemented the current study to primarily investigate the usefulness of performing a combined procedure of CTI ablation along with PVI using cryoballoon in patients referred to the participating centers for AFL ablation, with a documented non-predominant history of AF lasting at least $30 \mathrm{~s}$.

\section{2 | METHODS}

This was a prospective, multicenter, open-label, randomized study with two parallel arms that was designed to assess the benefits of $\mathrm{PVI}+\mathrm{CTI}(\mathrm{CTI}+)$ vs $\mathrm{CTI}$ alone (CTI) in the prevention of AF recurrence in patients with an AF history who were referred to the electrophysiology laboratories for typical AFL ablation.

Patients were considered eligible for entry into the trial if the following inclusion criteria were met: (i) diagnosis of typical AFL confirmed by electrocardiography (ECG) or 24-h Holter monitoring, with AFL defined as negative $F$ waves in the inferior territory, positive in V1, and negative in V6 lead, an F pattern with a characteristic "saw tooth" appearance, and an atrial cycle length comprised between 188 and 250 ms; with respect to patients arrhythmias, AF was neither the predominant (occasionally recorded) nor the targeted arrhythmia. The time duration spent by the patient in AFL should largely exceed that of in AF; (ii) at least one AF episode lasting over $30 \mathrm{~s}$ documented on either the ECG or Holter recording; (iii) effective anticoagulant therapy comprising either new oral anticoagulants like rivaroxaban or dabigatran, or vitamin $\mathrm{K}$ antagonists (VKA) with an international normalized ratio (INR) $\geq 2$ for at least 3 weeks; (iv) patient age between 18 and 75 years. Patients with contraindications to right-atrial catheterization or anticoagulant therapy were excluded from participation, as were those with predominant $\mathrm{AF}$ (or in $\mathrm{AF}$ at the screening/enrolment visit), known clotting disorders, or life expectancy $<24$ months.

The patients were screened after being referred to the electrophysiology laboratory for AFL ablation and only included in the trial after providing written informed consent.

The primary study endpoint was the recurrence of symptomatic or asymptomatic AF, documented on ECG or long-term Holter recording upon the 2-year follow-up (FU) beginning after the 12-week blanking period postablation, with $\mathrm{AF}$ recurrence defined as an atrial tachycardia episode of $>30 \mathrm{~s}$. Any episode of typical AFL $>30 \mathrm{~s}$ between Day 1 and Month 24 was considered AFL recurrence and a secondary study endpoint. The patients' quality of life was likewise assessed before and after the procedure, along with its evolution, the use of antiarrhythmic drugs, anticoagulants, and platelet inhibiting agents, and the rate of procedure-related complications, such as hematoma at the puncture site, thromboembolic events, and phrenic nerve palsy.

Prior to patient entry, we specified a 2-year recruitment period to enroll 170 subjects. Over the course of the study, however, owing to the much better outcome obtained using second-generation Advance cryoballoons, with 1-year AF recurrence rates of $20 \%$ instead of $30 \%-35 \%, 6,7$ a protocol amendment was forwarded to the national ethics committee requesting to reduce the total patient number to be recruited to 76 patients, ie, 38 per group. Based on an expected $55 \% \mathrm{AF}$ recurrence rate in the CTI group vs $25 \%$ in the $\mathrm{CTI}+$ group, a bilateral Chi-squared test would be able to detect a statistically significant between-group difference with an $80 \%$ statistical power and $5 \%$ risk level.

The study protocol, with its aforementioned amendment concerning the number of patients to be recruited, was approved by the national ethic committee and registered at clinicaltrials.gov under NCT 01521988

Eligible patients were randomized using a 1/1 ratio by block, with stratification by center, to allocate an equal number of patients to each ablation therapy: CTI alone for Group 1 and CTI+for Group 2. More blocks then necessary were created for any potential overrecruitment by a given investigator.

The RFA was performed under anatomic and electrophysiological guidance. Antiarrhythmic drugs were not systematically stopped before the procedure. Standardized procedures were employed, with the use of a quadripolar 6-F catheter positioned in the coronary sinus and a $4 \mathrm{~mm}$ tip irrigated RF catheter placed at the CTI. ${ }^{8-11}$ The RF ablation was conducted according to original research, with the main objective being to obtain a complete bidirectional isthmus conduction block $30 \mathrm{~min}(\mathrm{~min})$ after the last RF application via recording widely spaced double potentials along the 
ablation line, as well as inversion of the activation sequence opposite of the pacing site.

The procedure began with the CTI ablation, as described above. Thereafter, transseptal access was performed according to the centers' practices, potentially guided by transesophageal echocardiography (TEE). Standardized procedures were employed. ${ }^{12} \mathrm{~A}$ balloon catheter of $23-28 \mathrm{~mm}$ in diameter was positioned in the left atrium, and, then, applied at the PV ostium for ablation, with a minimum of two cryo-applications of at least 4 min carried out at each PV ostium. The procedural endpoint of the PVI was the disappearance of the PV potentials or an electrical dissociation between the $\mathrm{PV}$ and the atrium assessed by a circular octopolar catheter (Achieve ${ }^{\circledR}$, Medtronic Inc, Minneapolis, USA) inserted in the central lumen of the cryoballoon catheter. The remaining conduction gaps between the atrium and PV were ablated with the irrigated RF catheter used for CTI ablation.

During the hospital stay, cardiac rhythm monitoring was performed using continuous telemetry (scope) or 24-h Holter recording. AADs were continued for 3 months after the ablation as necessary, with the exception of amiodarone, which was discontinued in all cases postablation. Irrespective of the study arm, all patients received anticoagulant therapy 3 weeks before ablation that was maintained at least 3 months postablation. Antivitamin K (AVKs) agents were either maintained or replaced by heparin during the procedure. Managing new oral anticoagulants like rivaroxaban or dabigatran was left to the discretion of each center.

All patients were discharged home the day following the intervention. The patients were re-examined before discharge and at Weeks $12,26,52$, and 104 ( \pm 2 weeks) postprocedure, with a clinical examination, cardiology evaluation, 12-lead ECG, quality of life questionnaire (SF 36), medical treatment recording, and 7-day Holter monitoring performed. Documentation of AF or AFL during the first 12-week period postablation was not considered a true recurrence unless it persisted beyond this frame.

Only one new AFL ablation was permitted during the first 3 months postablation, irrespective of the group. In the presence of symptomatic AF recurrence despite medical treatment in the combined group after Week 26, a second PVI procedure was recommended.

Continuous variables were expressed as mean \pm standard deviation (SD) for normal data or median and interquartile range (IQR) for non-normal data, or counts and percentages for categorical variables. The analysis was carried out using the intent-to-treat method. Between-group comparisons were made using the Wilcoxon-MannWhitney test for categorical data and Fisher's exact $t$ test for qualitative data. Kaplan-Meier methodology was applied to determine the probability of $A F$ (and $A F L$ ) recurrence postablation, ignoring the 12-week blanking period postablation. Univariate Cox regression analyses were performed to determine the clinical predictors of AF occurrence.

In the absence of homoscedasticity, the Welch test was applied. All tests were two-sided, with a $P$-value $<0.05$ considered statistically significant. All analyses were performed using the R 3.5 .2 software (R Foundation for Statistical Computing).

\section{3 | RESULTS}

The patient characteristics are summarized in Table 1. During the 2-year recruitment period, 76 patients fulfilling the inclusion criteria were recruited, four of whom were lost to FU, for a total of 72 patients analyzed, ie, 36 per treatment arm. All patients had either failed AADs administration or experienced intolerable undesirable effects. The Group 1 patients $(n=36,65.6 \pm 9.7$ years, $72 \%$ male, $39 \%$ high blood pressure, $17 \%$ diabetic, and $8 \%$ heart failure New York Heart Association [NYHA] functional class $\geq 1$ ) were assigned to undergo CTI only, whereas the Group 2 patients $(n=36$, $62.1 \pm 8.6$ years, $83 \%$ male, $42 \%$ high blood pressure, $22 \%$ diabetic, and $28 \%$ heart failure) received the $\mathrm{CTI}+\mathrm{PVI}$ intervention. The baseline patient characteristics were roughly similar between both groups (Table 1).

At the time of the intervention, 19 patients in Group 1 and 18 in Group 2 presented with typical AFL, with two and four exhibiting $A F$, respectively (Table 2 ). In the latter, patients, cardioversion was performed before proceeding with ICT ablation. A normal sinus rhythm was obtained in all patients. Successful PVI was demonstrated in $100 \%$ of left common trunks (6/6), in $97 \%$ of left superior PVs (29/30), 100\% of left inferior PVs (30/30), 94\% of right superior PVs (34/36), and 89\% of right inferior PVs (32/36). One RF application was delivered to isolate the left upper PV in one patient, and the right lower PV in another one. Expectedly, the ablation procedure duration was shorter in Group 1 vs Group 2 (62.5 vs 109.5 min, respectively).

Overall, 72 patients were followed-up, with the recurrence of AF and other cardiac events analyzed at 1-year and 2-year postablation. The data are summarized in Table 3 and illustrated in Figure 1. At the study's end, 25 patients $(69 \%)$ reached the primary endpoint in Group 1 vs 12 (33\%) in Group 2, with the between-group difference being highly significant $(P=2.7 \mathrm{e}$ 0.04). Likewise, symptomatic AF occurred in 15 (Group 1) vs seven patients (Group 2), with a significant between-group difference as well (0.025). Due to the small sample size, no patient characteristics could be found as predictive of lack of AF recurrences after $\mathrm{CTI}$ alone (in particular, the introduction of AADs for occurrence of AF, which could have promoted later on, the development of AFL). The majority of the Group 1 patients (8/11 patients) without experiencing AF recurrences at the end of follow up were on AADs. Whereas patients under antiarrhythmic drugs and anticoagulants tended to be fewer in Group 1 vs Group 2 at 1 year (52\% vs 67\%, and 69\% vs $86 \%$ respectively), this difference was no longer present at 2 years follow-up. No significant differences were noted concerning the other secondary endpoints.

The combined ablation in comparison with $\mathrm{CTI}$ alone was associated with a reduction in the $\mathrm{HR}$ to $0.19(95 \% \mathrm{Cl}: 0.07 ; 0 / 43, P=9 \mathrm{e}-5)$.

No between-group differences with respect to the overall SF36 quality of life score were observed nor were there any significant differences recorded with respect to the different score subitems (data not shown). 


\begin{tabular}{|c|c|c|c|}
\hline Variable & $\begin{array}{l}\text { Flutter ablation } \\
\mathrm{n}=36\end{array}$ & Combined ablation $n=36$ & $\begin{array}{l}P \\
\text { value }\end{array}$ \\
\hline Age & $65.6(9.7)$ & $62.1(8.6)$ & 0.066 \\
\hline \multicolumn{4}{|l|}{ Gender } \\
\hline Male & $26(72 \%)$ & $30(83 \%)$ & \multirow[t]{2}{*}{ ns } \\
\hline Female & $10(28 \%)$ & $6(17 \%)$ & \\
\hline Body mass index & $28.5(6)$ & $28.2(5.5)$ & ns \\
\hline High blood pressure & $14(39 \%)$ & $15(42 \%)$ & ns \\
\hline Diabetes & $6(17 \%)$ & $8(22 \%)$ & ns \\
\hline Heart failure history & $3(8 \%)$ & $10(28 \%)$ & ns \\
\hline Stroke history & $3(8 \%)$ & $1(3 \%)$ & ns \\
\hline $\begin{array}{l}\text { Transient ischemic attack } \\
\text { history }\end{array}$ & $1(3 \%)$ & $2(6 \%)$ & ns \\
\hline Distal embolism history & $2(6 \%)$ & $0(0 \%)$ & ns \\
\hline Lower limb arterial disease & $1(3 \%)$ & $1(3 \%)$ & ns \\
\hline Carotid atheroma & $0(0 \%)$ & $1(3 \%)$ & ns \\
\hline Paroxysmal AF & $29(81 \%)$ & $25(69 \%)$ & ns \\
\hline Antiarrhythmic treatment & & & ns \\
\hline Amiodarone & $12(33 \%)$ & $18(50 \%)$ & \\
\hline Sotalol & $2(6 \%)$ & $0(0 \%)$ & \\
\hline Ic antiarrhythmic drug & $9(25 \%)$ & $9(25 \%)$ & \\
\hline la antiarrhythmic drug & $2(6 \%)$ & $1(3 \%)$ & \\
\hline
\end{tabular}

TABLE 1 Population characteristics

Abbreviation: AF, atrial fibrillation.

TABLE 2 Procedures, duration, and outcomes per group

\begin{tabular}{lll} 
& $\begin{array}{l}\text { Flutter ablation } \\
\mathrm{n}=36\end{array}$ & $\begin{array}{l}\text { Combined } \\
\text { ablation } \mathrm{n}=36\end{array}$ \\
$\begin{array}{l}\text { Heart rhythm at the } \\
\text { beginning }\end{array}$ & & \\
$\begin{array}{l}\text { Sinus rhythm } \\
\text { Typical atrial flutter }\end{array}$ & $15(42 \%)$ & $14(39 \%)$ \\
Atrial fibrillation & $2(6 \%)$ & $18(50 \%)$ \\
Heart rhythm at the end & $4(11 \%)$ \\
Sinus rhythm & $34(94 \%)$ & $35(97 \%)$ \\
Unknown & $2(6 \%)$ & $1(3 \%)$ \\
\hline Duration of procedure (min) & $62.5[23 ; 146]$ & $109.5[38 ; 227]$ \\
\hline $\begin{array}{l}\text { Total radiofrequency ablation } \\
\text { time (s) }\end{array}$ & $727[69 ; 2797]$ & $472[61 ; 2813]$ \\
\hline Bidirectional complete & & \\
isthmic block obtained & & $0(0 \%)$ \\
No & $2(6 \%)$ & $34(94 \%)$ \\
Yes & $33(92 \%)$ & $2(6 \%)$ \\
Unknown & $1(3 \%)$ & \\
\hline
\end{tabular}

Note: Quantitative data are expressed as median [min;max]. Qualitative data are expressed as rates (percentage).

In the postintervention period, one Group 2 patient had a small pericardial effusion that did not require any drainage. One patient in each group developed a first-degree atrioventricular block, and another Group 1 patient exhibited a third-degree atrioventricular block resulting in pacemaker implantation. Three Group 2 patients exhibited phrenic nerve palsy that spontaneously disappeared within a few weeks. Two Group 2 patients displayed a groin hematoma that did not require any intervention. The number of hospitalization postablation was roughly similar between both groups, as was the number of new ablations required (Table 4). One Group 2 patient developed pulmonary vein stenosis following PVI, which was deemed not clinically relevant. One patient in each group developed stroke during the follow up period ( 9 and 20 months after the procedure).

\section{DISCUSSION}

It must be emphasized once more that several studies have clearly revealed that, even with AFL being eliminated by means of CTI ablation, the postprocedural incidence of new-onset $A F$ can reach $\geq 55 \%$ at 1-year postablation. ${ }^{13}$ In AFL patients, associated AF has also been found to be a strong predictive factor of new-onset $A F$ following CTI ablation. ${ }^{14}$ Owing to these high AF rates, more comprehensive interventions have been developed over the last decade. In their randomized pilot study, Mohantly et al enrolled 216 patients with isolated AFL, yet no prior AF history, who were randomly assigned to undergo either CTI ablation alone (Group 1) or CTI+PVI ablation (Group 2). ${ }^{15}$ At $18 \pm$ 6-month FU, 60\% Group 1 patients were shown to be arrhythmia-free vs $71 \%$ Group 2 patients, with a statistically significant $(P=.044)$ between-group difference. The 3-year follow-up data of the Prevent AF I study involving $50 \mathrm{AFL}$ 
TABLE 3 Probability of arrhythmia events over the 2-year study period

\begin{tabular}{|c|c|c|c|c|}
\hline & & Group $1 \mathrm{n}=36$ & Group $2 n=36$ & $\begin{array}{l}P \\
\text { value }\end{array}$ \\
\hline \multirow[t]{3}{*}{ 30-s AF } & $\begin{array}{l}\text { Overall } \\
\text { frequency }\end{array}$ & $25(69 \%)$ & 12 (33\%) & $2.7 e-04$ \\
\hline & 1 year & $0.73[0.53 ; 0.84]$ & $0.23[0.08 ; 0.36]$ & \\
\hline & 2 years & $0.73[0.53 ; 0.84]$ & $0.38[0.18 ; 0.53]$ & \\
\hline \multirow[t]{3}{*}{ 1-min $A F$} & $\begin{array}{l}\text { Overall } \\
\text { frequency }\end{array}$ & $25(69 \%)$ & 12 (33\%) & $2.7 e-04$ \\
\hline & 1 year & $0.73[0.53 ; 0.84]$ & $0.23[0.08 ; 0.36]$ & \\
\hline & 2 years & $0.73[0.53 ; 0.84]$ & $0.38[0.18 ; 0.53]$ & \\
\hline \multirow[t]{3}{*}{ Symptomatic AF } & $\begin{array}{l}\text { Overall } \\
\text { frequency }\end{array}$ & $15(42 \%)$ & $7(19 \%)$ & .025 \\
\hline & 1 year & $0.4[0.22 ; 0.55]$ & $0.11[0 ; 0.21]$ & \\
\hline & 2 years & $0.44[0.24 ; 0.58]$ & $0.22[0.06 ; 0.36]$ & \\
\hline \multirow[t]{3}{*}{ Persistent AF } & $\begin{array}{l}\text { Overall } \\
\text { frequency }\end{array}$ & $5(14 \%)$ & $8(22 \%)$ & .32 \\
\hline & 1 year & $0.06[0 ; 0.14]$ & $0.14[0.02 ; 0.25]$ & \\
\hline & 2 years & $0.17[0.02 ; 0.29]$ & $0.25[0.08 ; 0.39]$ & \\
\hline \multirow[t]{3}{*}{ Typical AFL } & $\begin{array}{l}\text { Overall } \\
\text { frequency }\end{array}$ & $6(17 \%)$ & $6(17 \%)$ & .886 \\
\hline & 1 year & $0.15[0.02 ; 0.26]$ & $0.14[0.02 ; 0.25]$ & \\
\hline & 2 years & $0.19[0.04 ; 0.31]$ & $0.17[0.04 ; 0.29]$ & \\
\hline \multirow[t]{3}{*}{ Atypical AFL } & $\begin{array}{l}\text { Overall } \\
\text { frequency }\end{array}$ & $4(11 \%)$ & $5(14 \%)$ & .74 \\
\hline & 1 year & $0.09[0 ; 0.18]$ & 0.08 [0;0.17] & \\
\hline & 2 years & $0.13[0 ; 0.24]$ & $0.16[0.02 ; 0.28]$ & \\
\hline \multirow[t]{3}{*}{ Atrial tachycardia } & $\begin{array}{l}\text { Overall } \\
\text { frequency }\end{array}$ & 4 (11\%) & $3(8 \%)$ & .663 \\
\hline & 1 year & $0.09[0 ; 0.18]$ & $0.09[0 ; 0.17]$ & \\
\hline & 2 years & $0.13[0 ; 0.24]$ & 0.09 [0;0.17] & \\
\hline
\end{tabular}

Abbreviations: $A F$, atrial fibrillation; $A F L$, atrial flutter.

*Non-parametric log-rank test. patients were published in 2018 by Romanov et al. ${ }^{16}$ Similarly, the study results revealed that in patients with typical AFL, adding PVI to $C T I$ ablation turned out to be safe and effective in terms of AF freedom as compared with CTI alone. Indeed, at 3-year FU, freedom from any tachyarrhythmia was observed in $48 \% \mathrm{PVI}+\mathrm{CTI}$ patients as compared with $20 \%$ CTI-only patients ( $P=.01$ ). Recently, in 2019 , Koeber et al have published a meta-analysis involving four randomized trials with similar study designs. These authors also sought to better understand the effect of combined CTI+PVI vs CTI alone in patients scheduled for AFL ablation. ${ }^{17}$ Considering the 316 randomized patients in the analysis, AF freedom at 1-year postablation was significantly higher in the combined treatment group vs $\mathrm{CTI}$ ablation alone.

Based on the hypothesis that AF is chiefly initiated by triggers originating from the $\mathrm{PVs},{ }^{18}$ our data have further confirmed these aforementioned observations, ie, the clinical usefulness of combining $\mathrm{PVI}+\mathrm{CTI}$ in $\mathrm{AFL}$ patients with at least $1 \mathrm{AF}$ episode, despite the latter being not the predominant arrhythmia. In our study, AF recurrence was significantly less common when PVI was implemented at the time of CTI for typical AFL, in line with published data. In the Mohantly et al study, at $18 \pm 6$-month FU, about $40 \%$ patients developed $\mathrm{AF}$ in the $\mathrm{CTI}$-only group vs $29 \%$ in the $\mathrm{CTI}+$ group. ${ }^{15} \mathrm{After}$ carefully analyzing these published reports, we conclude that in patients with prior AF at the time of CTI ablation, as in our study population, or in those without prior AF, as in the Mohantly study ${ }^{15}$ quite similar AF incidence rates (about 30\%) were reported at 18-24-month FU in the combined treatment groups. These incidence rates, however, differed in the $\mathrm{CTI}$-only groups. Whereas $70 \% \mathrm{AF}$ recurrence rates were recorded in our study involving patients with prior $\mathrm{AF}$, the rates of new onset AF were only $40 \%$ in the Mohantly study involving AF-naïve patients. ${ }^{15}$ The benefit of adding PVI to CTI was, thus, more convincing in our study. This differing outcome may possibly be explained by the observation that in our study, patients were required to display at least one prior $30 \mathrm{~s}$ AF episode to be eligible for study entry, whereas the Mohantly et al study recruited only AF-naïve patients.

Only limited data are available concerning the overall cost effectiveness of a combined $\mathrm{PVI}+\mathrm{CTI}$ approach vs a sequential approach. 


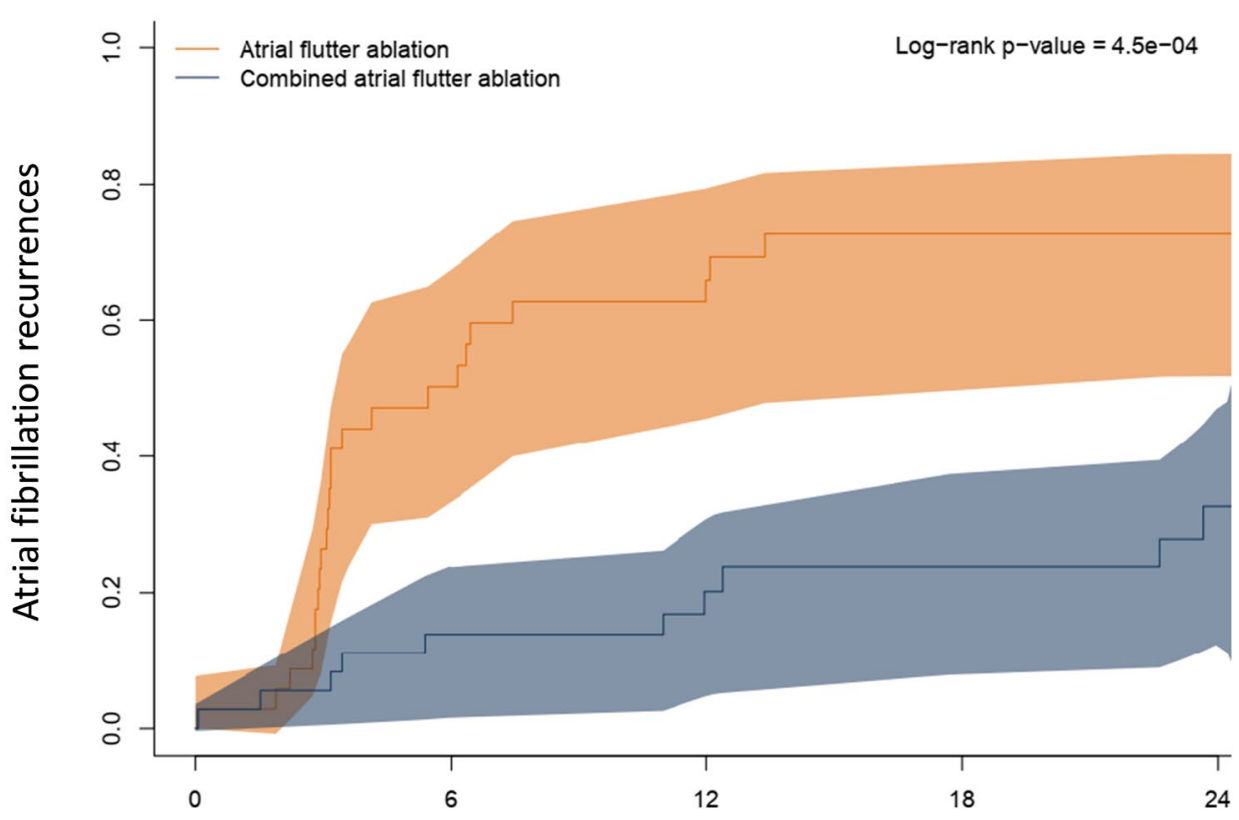

Follow-up in months

FIGURE 1 Survival curves regarding atrial fibrillation recurrences during follow-up

\begin{tabular}{|c|c|c|c|}
\hline Complication & $\begin{array}{l}\text { Flutter ablation } \\
n=36\end{array}$ & $\begin{array}{l}\text { Combined ablation } \\
n=36\end{array}$ & $\begin{array}{l}P \\
\text { value }\end{array}$ \\
\hline \multicolumn{4}{|l|}{ Pericardial complication } \\
\hline None & $36(100 \%)$ & 35 (97\%) & \\
\hline Effusion without evacuation & 0 & $1(3 \%)$ & \\
\hline \multicolumn{4}{|l|}{ Atrioventricular block } \\
\hline None & $34(94 \%)$ & 35 (97\%) & \\
\hline AVB I & $1(3 \%)$ & $1(3 \%)$ & \\
\hline AVB III & $\begin{array}{l}1(3 \%), \text { pacemaker } \\
\text { implantation }\end{array}$ & 0 & \\
\hline Phrenic complication & 0 & $3(8 \%)$ & .079 \\
\hline Transient ischemic attack & 0 & 0 & \\
\hline Stroke & $1(3 \%)$ & $1(3 \%)$ & .998 \\
\hline Hospitalization & $23(64 \%)$ & $15(42 \%)$ & .173 \\
\hline New ablation & $8(22 \%)$ & $6(17 \%)$ & .585 \\
\hline Typical flutter ablation & 3 & 2 & \\
\hline Ablation of atrial fibrillation & 5 & 5 & \\
\hline Ablation of atrial tachycardia & 0 & 1 & \\
\hline Pulmonary venous stenosis & 0 & $1(3 \%)$ & .308 \\
\hline
\end{tabular}

TABLE 4 Occurrence of complications per treatment group

Abbreviation: AVB, atrioventricular block.

${ }^{*}$ Non-parametric log-rank.

Referring to the 3-year FU data of Prevent AF I study, Romanov et al reported overall lower costs and procedural risks with a combined ablation procedure. ${ }^{16}$ These benefits were driven by a higher atrial arrhythmia recurrence rate at 3 years (80\%), as well as a significantly higher rate of repeat procedures and hospitalizations in the CTI-only group. Contrarily, no significant differences in major complications requiring interventions were recorded with the combined procedure.

Further larger-scale, randomized studies including cost-efficacy analyses are still needed to better guide appropriate patient selection for ablation procedures in AFL management. If the combined approach would prove to be cost-effective in patients with "pure" 
AFL in such larger-scale studies, we could then assume that a combined procedure would indeed be preferable as first-line therapy in patients with prior AF episodes.

The question as to whether AF and AFL could be initiated by the same electrophysiological triggers has previously been raised by numerous experts. ${ }^{19}$ A close pathophysiological relationship between AFL and AF is backed up by experimental studies and clinical data. ${ }^{20}$ As further outlined in the nicely written review paper by Waldo $A L, A F$ is usually required for the development of a functional block between the venae cavae. ${ }^{19}$ This latter, in turn, seems to be necessary to enable a classical AFL to develop. According to Wazni et al, AF usually precedes the onset of AFL. Indeed, recent studies have demonstrated the key role that PVs play in initiating $A F^{21}$ As proposed by Roithinger et al, ${ }^{22}$ and in line with Wazni et al, ${ }^{21} \mathrm{PV}$ triggers initiate AF, transforming itself subsequently into AFL. According to Roithinger et al, PV triggers may be responsible for the development of AFL, under appropriate circumstances.

In agreement with this line of thoughts, the aforementioned publications, and the Navarrete et al's report, ${ }^{23}$ our data likely confirm that it is rather logical to perform PVI in patients scheduled to undergo CTI for clinical AFL. In line with the conclusions drawn by Navarrete et al, that clinicians should be made aware of the robust link that exists between AFL and AF. That is to say, when CTI ablation is performed to interrupt AFL, it may be useful to assume that procedural PVI be carried out at the same time, at least in some selected patients, notably those with an AF history. The ultimate aim of this combined procedure would be to definitively diminish the high thromboembolic risk, even in the absence of clinically relevant $\mathrm{AF}$ We should however underline that the potential increased risk of complications associated with PVI is likely the main reason physician would refrain to perform this procedure in this patient population. Unfortunately, the small patients sample size of our study precluded drawing any conclusion regarding complications. In any event, the still significant long-term risk of recurrent $A F$ in this patient group is an incentive to adhere to general anticoagulation recommendations, regardless of the ablation strategy chosen and the presumed rhythm outcome, ie, high stroke risk patients should continue anticoagulation.

The results of this study should be interpreted in light of several methodological limitations, the major one being the limited number of patients per treatment group. Owing to constraints imposed by the ethic committee that requested patients to have experienced at least one $30 \mathrm{~s}$ AF episode to be eligible for study entry, recruiting sufficient patients proved very challenging, and a protocol amendment was thus issued. Keeping this limitation in mind, it is essential that our study results be further confirmed in larger-sized randomized comparative trials. Another limitation to be pointed out is that maintenance of AADs along the course of the study may have masked the presence of manifest $A F$, particularly in patients with persistent AF before the procedure. The patients most likely to benefit from this combined approach must still be better defined, in addition to in-depth cost-benefit analysis.

\section{5 | CONCLUSIONS}

The results of this prospective randomized study likely indicate that $\mathrm{PVI}$ in conjunction with $\mathrm{CTI}$ in AFL patients with history of nonpredominant AF scheduled for AFL ablation significantly decreases the incidence of $A F$ recurrence at both 1- and 2-year postablation periods.

\section{ACKNOWLEDGMENTS}

This work was supported by a grant from Medtronic. The authors thank Dr Gabrielle Cremer for her help in the manuscript writing.

\section{CONFLICT OF INTEREST STATEMENT}

FA is consultant for and received lecture fees from Boston Scientific, Medtronic, and Microport CRM. AS received honoraria from Microport CRM. NC is consultant for and received lecture fees from Medtronic. PD received research grants and honoraria from Boston Scientific, Abbott, Microport CRM, and Medtronic. SB is consultant for Medtronic, Boston Scientific, Microport CRM, and Zoll. The other authors declare no conflicts of interest. The protocol for this research has been approved by a suitable constituted Ethics Committee of the institution under the approval number 2011-A00806-35, on July 21, 2011 and it conforms to the provisions of the Declaration of Helsinki. The ClinicalTrial identifier of this study is NCT01521988

\section{Data Availability Statement}

The data that support the findings of this study are available from the corresponding author upon reasonable request.

\section{ORCID}

Frédéric Anselme iD https://orcid.org/0000-0002-2130-9790

\section{REFERENCES}

1. Cauchemez B, Haissaguerre M, Fischer B, Thomas O, Clementy J, Coumel P. Electrophysiological effects of catheter ablation of inferior vena cava-tricuspid annulus isthmus in common atrial flutter. Circulation. 1996;93(2):284-94

2. Pérez FJ, Schubert CM, Parvez B, Pathak V, Ellenbogen KA, Wood MA. Long-term outcomes after catheter ablation of cavo-tricuspid isthmus dependent atrial flutter: a meta-analysis. Circ Arrhythm Electrophysiol. 2009;2(4):393-401

3. Bertaglia E, Zoppo F, Bonso A, Proclemer A, Verlato R, Corò L, et al. Long term follow up of radiofrequency catheter ablation of atrial flutter: clinical course and predictors of atrial fibrillation occurrence. Heart. 2004;90(1):59-63.

4. Da Costa A, Romeyer C, Mourot S, Messier M, Cerisier A, Faure E, et al. Factors associated with early atrial fibrillation after ablation of common atrial flutter. A single centre prospective study. Eur Heart J. 2002;23(6):498-506.

5. Linhart M, Bellmann B, Mittmann-Braun E, Schrickel JW, Bitzen A, Andrié R, et al. Comparison of cryoballoon and radiofrequency ablation of pulmonary veins in 40 patients with paroxysmal atrial fibrillation: a case-control study. J Cardiovasc Electrophysiol. 2009;20(12):1343-8.

6. Chierchia G-B, Di Giovanni G, Ciconte G, de Asmundis C, Conte G, Sieira-Moret J, et al. Second-generation cryoballoon 
ablation for paroxysmal atrial fibrillation: 1-year follow-up. Europace. 2014;16(5):639-44.

7. Metzner A, Reissmann B, Rausch P, Mathew S, Wohlmuth P, Tilz $\mathrm{R}$, et al. One-year clinical outcome after pulmonary vein isolation using the second-generation 28-mm cryoballoon. Circ Arrhythm Electrophysiol. 2014;7(2):288-92.

8. Anselme F, Saoudi N, Poty H, Douillet R, Cribier A. Radiofrequency catheter ablation of common atrial flutter: significance of palpitations and quality-of-life evaluation in patients with proven isthmus block. Circulation. 1999;99(4):534-40.

9. Anselme F, Savouré A, Cribier A, Saoudi N. Catheter ablation of typical atrial flutter: a randomized comparison of 2 methods for determining complete bidirectional isthmus block. Circulation. 2001;103(10):1434-9.

10. Hsieh M-H, Tai C-T, Chiang C-E, Tsai C-F, Yu W-C, Chen Y-J, et al. Recurrent atrial flutter and atrial fibrillation after catheter ablation of the cavotricuspid isthmus: a very long-term follow-up of 333 patients. J Interv Card Electrophysiol. 2002;7(3):225-31.

11. Jaïs $P$, Shah DC, Haïssaguerre M, Hocini M, Garrigue S, Le Metayer $\mathrm{P}$, et al. Prospective randomized comparison of irrigated-tip versus conventional-tip catheters for ablation of common flutter Circulation. 2000;101(7):772-6.

12. Chun K-RJ, Schmidt B, Metzner A, Tilz R, Zerm T, Köster I, et al. The "single big cryoballoon" technique for acute pulmonary vein isolation in patients with paroxysmal atrial fibrillation: a prospective observational single centre study. Eur Heart J. 2009;30(6):699-709.

13. Mittal S, Pokushalov E, Romanov A, Ferrara M, Arshad A, Musat $D$, et al. Long-term ECG monitoring using an implantable loop recorder for the detection of atrial fibrillation after cavotricuspid isthmus ablation in patients with atrial flutter. Heart Rhythm. 2013;10(11):1598-604.

14. Celikyurt U, Knecht S, Kuehne M, Reichlin T, Muehl A, Spies F, et al. Incidence of new-onset atrial fibrillation after cavotricuspid isthmus ablation for atrial flutter. Europace. 2017;19(11):1776-80.

15. Mohanty S, Natale A, Mohanty P, Di biase L, Trivedi C, Santangeli P, et al. Pulmonary vein isolation to reduce future risk of atrial fibrillation in patients undergoing typical flutter ablation: results from a randomized pilot Study (REDUCE AF). J Cardiovasc Electrophysiol. 2015;26(8):819-25.

16. Romanov A, Pokushalov E, Bayramova S, Ponomarev D, Shabanov V, Losik D, et al. Prophylactic pulmonary vein isolation during isthmus ablation for atrial flutter: three-year outcomes of the PREVENT AF I study. J Cardiovasc Electrophysiol. 2018;29(6):872-8.

17. Koerber SM, Turagam MK, Gautam S, Winterfield J, Wharton JM, Lakkireddy D, et al. Prophylactic pulmonary vein isolation during cavotricuspid isthmus ablation for atrial flutter: a meta-analysis. Pacing Clin Electrophysiol. 2019;42(5):493-8.

18. Haïssaguerre M, Jaïs P, Shah DC, Takahashi A, Hocini M, Quiniou G, et al. Spontaneous initiation of atrial fibrillation by ectopic beats originating in the pulmonary veins. N Engl J Med. 1998;339(10):659-66.

19. Waldo AL. Mechanisms of atrial flutter and atrial fibrillation: distinct entities or two sides of a coin? Cardiovasc Res. 2002;54(2):217-29.

20. Waldo AL, Feld GK. Inter-relations hips of atrial fibrillation and atrial flutter mechanisms and clinical implications. J Am Coll Cardiol. 2008;51(8):779-86.

21. Wazni O, Marrouche NF, Martin DO, Gillinov AM, Saliba W, Saad E, et al. Randomized study comparing combined pulmonary vein-left atrial junction disconnection and cavotricuspid isthmus ablation versus pulmonary vein-left atrial junction disconnection alone in patients presenting with typical atrial flutter and atrial fibrillation. Circulation. 2003;108(20):2479-83.

22. Roithinger FX, Karch MR, Steiner PR, SippensGroenewegen A, Lesh MD. Relationship between atrial fibrillation and typical atrial flutter in humans: activation sequence changes during spontaneous conversion. Circulation. 1997;96(10):3484-91.

23. Navarrete A, Conte F, Moran M, Ali I, Milikan N. Ablation of atrial fibrillation at the time of cavotricuspid isthmus ablation in patients with atrial flutter without documented atrial fibrillation derives a better long-term benefit. J Cardiovasc Electrophysiol. 2011;22(1):34-8.

How to cite this article: Anselme F, Savouré A, Clémenty N, Cesari O, Pavin D, Jesel L, et al. Preventing atrial fibrillation by combined right isthmus ablation and cryoballoon pulmonary vein isolation in patients with typical atrial flutter: PAFCRIOBLAF study. J Arrhythmia. 2021;37:1303-1310. https:// doi.org/10.1002/joa3.12626 